\title{
Caracterización de los cativales (Prioria copaifera Grisebach), presentes en el río León, departamento del Chocó
}

\section{Characterization of the cativales (Prioria copaifera Grisebach), present in the León River, Chocó Department}

\section{Romni Romaña-Hurtado*}

\section{Resumen}

Se describe la estructura y diversidad florística en el bosque de cativo del municipio de Riosucio, departamento del Chocó, Colombia. El muestreo se realizó en el corregimiento de Tumaradó, donde se establecieron dos parcelas de 0,25 ha $(50 \mathrm{~m}$ $x 50 \mathrm{~m}$ ). En ellas se delimitaron y se registraron las especies presentes y se midió el diámetro a la altura del pecho (DAP) de todos los individuos $\geq 10 \mathrm{~cm}$. La estructura del bosque estudiado presenta una distribución diamétrica en forma de " $J$ " invertida, la cual expresa la dinámica del bosque; también se elaboró un perfil esquemático. En total se identificaron 16 especies, pertenecientes a 16 géneros y 14 familias, siendo la especie Prioria copaifera Griseb., con el valor más alto de importancia estructural debido a su determinante área basal y su carácter gregario y dominante. De acuerdo con los índices de Margalef, Shannon y riqueza, la diversidad de especies fue significativamente menor en la unidad de muestro 1; en contraste, el índice de Simpson no mostró diferencias significativas entre las unidades de muestreo.

Palabras clave: Bosque de cativo, Distribución horizontal, Estratificación vertical, Riosucio.

\begin{abstract}
Structure and floristic diversity were studied in the cativo forests in Tumaradó, Riosucio. The sampling procesa was performed by establishing two 25 hectare $(50 \mathrm{~m} \times 50 \mathrm{~m})$ for the location. For both forests, botanical determination and record were taken for each species. Also diameter at breast height (DBH) were measured for all trees thicker than $10 \mathrm{~cm}$. Studied forests showns a diametric distribution of inverted " $J$ ". Also a schematic profile of forest. Being the species Prioria copaifera Griseb, with the highest value of structural importance because of its decisive
\end{abstract}

* Investigador Asociado, Instituto de Investigaciones Ambientales del Pacífico (IIAP), Quibdó, Colombia. e-mail: rromanaCiiap.org.co 
basal area and gregarious and dominant character. In accordance with the Margalef and Shannon indices, the richness, species diversity were significantly lower in unit 1; in contrast, Simpson index showed no significant difference between the sampling units.

Keywords: Forest of cativo, Horizontal distribution, Riosucio, Vertical stratification.

\section{Introducción}

La gran riqueza de especies de plantas del Chocó Biogeográfico y su alto endemismo ha sido ampliamente reconocida (Gentry 1982, Forero y Gentry 1989). Sin embargo, también se ha descrito la existencia de asociaciones vegetales cuya composición florística tiende a formar masas homogéneas de baja diversidad (Suárez et al. 1984, Rangel y Lowy 1993, del Valle 1996) donde existe alta abundancia de individuos de pocas especies; esta condición puede ser explicada por ejemplo, por la poca tolerancia de muchas especies arbóreas a los períodos de anegamiento, condiciones ambientales y edáficas diferentes (Hart 1990, Richards 1996, Duivenvoorden 1996, Marqués y Joly 2000, López y Kursar 2007).

En este sentido, algunas de estas asociaciones han sido denominadas cativales, las cuales se localizan al noroccidente de Colombia específicamente en los ríos Atrato, León y Nechí entre los departamento de Chocó y Antioquia y cerca de la ciudad de Santa Marta (López y Montero 2006), sobre llanuras aluviales en diferentes paisajes fisiográficos, y son consideradas únicas entre las comunidades tropicales, por su relativa homogeneidad, adecuada estructura, capacidad de regeneración y potencial facilidad de manejo, apreciable crecimiento volumétrico, productividad anual en biomasa, extensión y disponibilidad de aprovechamiento (Linares 1988). Además, poseen gran importancia ecológica y comercial por ofrecer diversos hábitat y medios alimenticios a innumerables individuos biológicos que conforman una cadena trófica compleja. Los bosques de cativo de acuerdo con Holdridge (1979) son asociaciones que se encuentran en suelo fértil, sobre llanuras aluviales periódicamente inundadas por agua dulce, en el piso basal tropical húmedo, cuya vegetación primaria es un bosque donde esta especie domina, formado por aproximadamente 60 especies arbóreas, 15 de las cuales representan el 95\% siendo una de las más representativas Carapa guianensis, Virola sp. y Lecythis sp., del total de individuos, pertenecientes a las familias Fabaceae, Caesalpinaceae, Bombacaceae y Sterculiaceae. En este sentido, la cantidad de biomasa que reporta los cativales alcanza 1.189 ton/ha cifra que lo pone entre los ecosistemas boscosos de más alta productividad biológica en el mundo tropical y cuyo volumen maderable en pie es de $547 \mathrm{~m}^{3} /$ ha (Golley 1969). De este modo, su alto valor de importancia permite elevados valores en áreas basales por encima de $55 \mathrm{~m}^{2} / \mathrm{ha}$.

En las últimas décadas la creciente demanda por su madera ha generado una presión por causas antrópicas, lo que la ha reducido drásticamente (Veja et al. 2005), teniendo presente que estos ecosistemas han sido los mayores productores de maderas tropicales en Colombia (Suárez et al. 1984, Del Valle 2005). Esto ha ocasionado a que el cativo (Prioria copaifera) fuera categorizado como una especie en peligro (EN) para Colombia (López y Montero 2006). Por todo lo anterior, con esta investigación se pretende sentar las bases desde el punto de vista ecológico y socioeconómico que garantice la sostenibilidad de estas comunidades forestales en el tiempo y el espacio. El presente estudio buscó caracterizar la estructura poblacional de los bosques de cativo, así como riqueza, índice de valor de importancia y diversidad de especies vegetales asociadas con el río León, corregimiento de Tumaradó municipio de Riosucio, Chocó, Colombia. 


\section{Bioetnia Volumen 14, 2017}

\section{Descripción del área de muestreo}

La presente investigación se llevó a cabo en

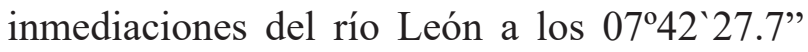
de latitud norte y $77^{\circ} 01^{\prime} 18.4^{\prime}$ ” en jurisdicción del corregimiento de Tumaradó, municipio de Riosucio, Chocó, Colombia, con una altura de $11 \mathrm{msnm}$, el cual presenta una temperatura anual que oscila entre $24^{\circ} \mathrm{C} \mathrm{y} 28^{\circ} \mathrm{C}$ y una precipitación entre 2.000 y 5.000 mm/año (Figura 1). Según el sistema de clasificación de Holdridge para Colombia el sitio corresponde a bosque húmedo tropical y bosque muy húmedo tropical (Escobar y Vásquez 1987). Los suelos del catival poseen texturas que varían de finas a medias, estructuras rocosas, pobremente drenados, profundidad efectiva media, hidromórficos, pegajosos, plásticos y grises; son suelos inundables de mediana a buena fertilidad, con adecuadas cantidades de calcio, magnesio y potasio, $\mathrm{pH}$ entre 5,5 y 6,5, bajo contenido de fósforo asimilable; en general, son suelos minerales con bajo contenido de materia orgánica. Son poco evolucionados, en su mayoría

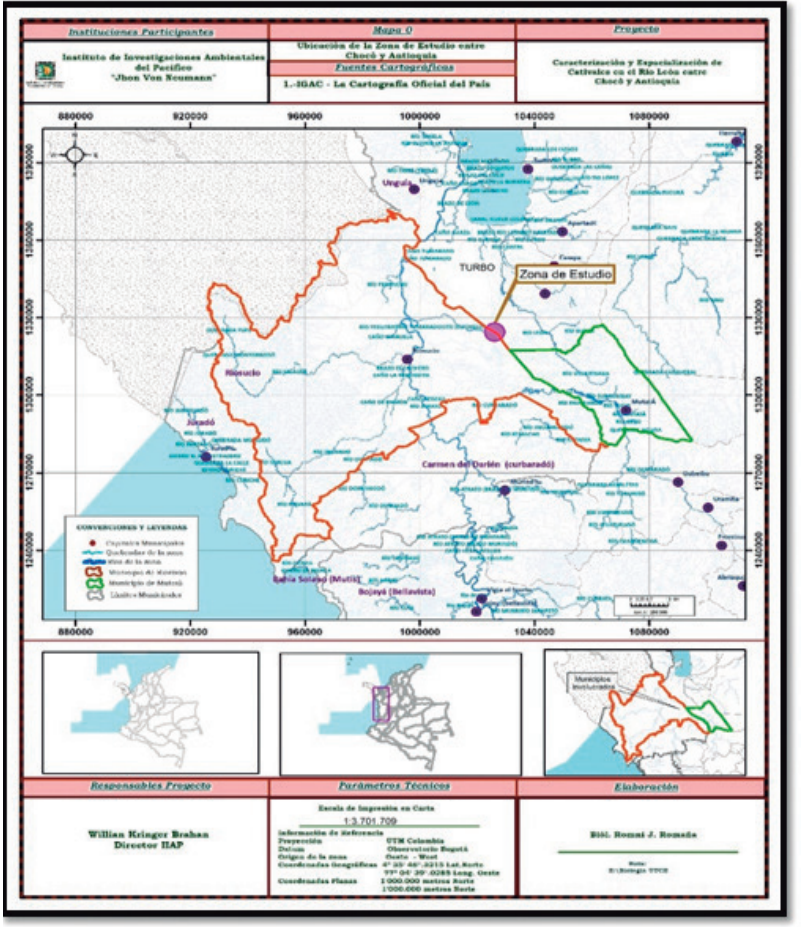

Figura 1. Ubicación de la zona de estudio.
Inceptisoles (Tropaquets y Eutropepts) y Entisoles (Tropaquents, Tropofluvents y Fluvaquents) (IGAC 1984).

\section{Métodos}

El trabajo de campo se llevó a cabo a través de un muestreo sistemático, en el cual se tuvo en cuenta algunos parámetros, descritos de la siguiente manera:

Procedimiento de muestreo y medición de variables. Se establecieron cuatro unidades de muestreo (UM) de $50 \mathrm{~m}$ x $50 \mathrm{~m}(0,25$ ha inventariada) cada una de las cuales fueron subdivididas en cuadrantes de $25 \times 25(0,0625 \mathrm{ha})$, las cuales se trazaron con ayuda de una brújula y delimitadas a través de estacas de madera. Cada cuadrante se localizó mediante coordenadas cartesianas (x, y), de acuerdo con lo sugerido por Hubbell y Foster (1983) y Dallmeier (1992) para bosques tropicales. Se censaron todos los individuos con diferentes hábitos de crecimiento, haciendo énfasis para el caso del arbolado adulto todos los enraizados dentro de las UM que presentaron un tronco definido con diámetros a 1,30 $\mathrm{m}$ de altura con DAP $\geq 10 \mathrm{~cm}$. A cada uno se le registró su identidad taxonómica, altura total y comercial de acuerdo con Dawkins (1958). Consecutivamente al censo, se realizó la recolecta por duplicado de ejemplares botánicos de cada morfoespecie, los cuales fueron procesados (Lot y Chiang 1986) e identificados taxonómicamente en el herbario de la Universidad Tecnológica del Chocó (UTCH), Quibdó, Colombia.

Cálculo de atributos dasométricos e índices estructurales. Con objeto de describir la estructura vertical y horizontal de las UM, se elaboraron histogramas de frecuencia por clase de altura y diámetro: la información del DAP de los individuos censados se organizó por intervalos de $10 \mathrm{~cm}$ (10 a 19,9, 20 a 29,9 y así sucesivamente), para la distribución horizontal; de igual modo, para el caso de las alturas relacionada con la distribución 


\section{Cativales en el río León. $\mathbf{R}$ Romaña-Hurtado}

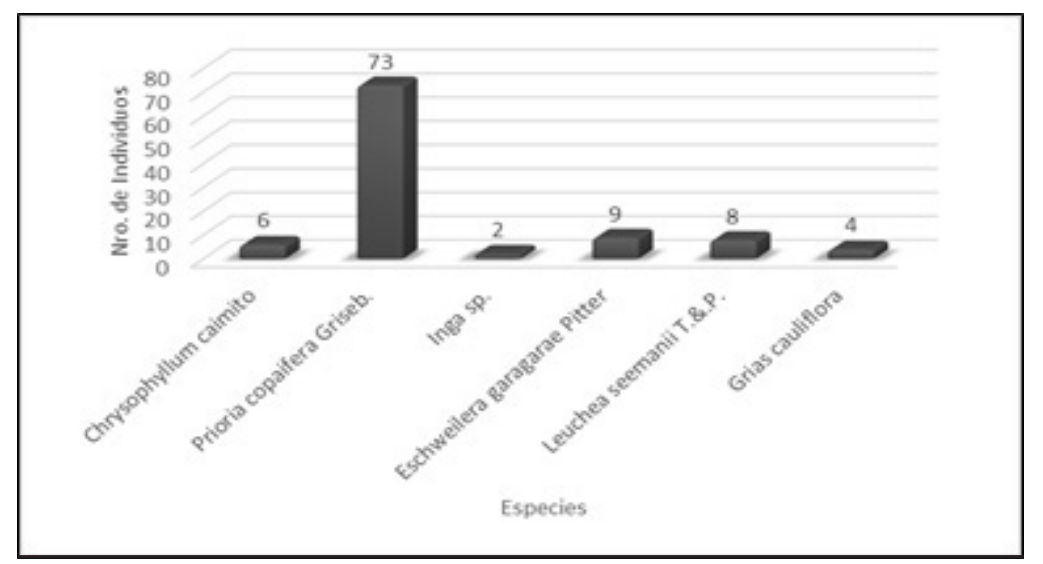

Figura 2. Especies más abundantes en las unidades de muestreo.

vertical se tuvo en cuenta una amplitud de $3 \mathrm{~cm}$ entre intervalos. Este análisis se llevó a cabo conjuntando para todos los valores de las 2 UM. De igual manera, la valoración estructural se hizo a través de índices que expresan la ocurrencia de las especies, lo mismo que su importancia ecológica dentro del ecosistema; es el caso de las abundancias, frecuencias y dominancias, cuya suma relativa genera es el índice de valor de importancia (IVI) (Curtis y McIntosh 1951).

Medidas de la diversidad de especies. A partir de la información obtenida para cada UM, se elaboró una tabla con respecto al número de especies vegetales encontradas para superficie de muestreo determinada (Greig-Smith 1983). De esta forma, para el caso de la riqueza de especies(D) se calculó mediante el índice de Margalef (1977), equidad de acuerdo con Shannon-Wiener, y dominancia con Simpson (Fisher et al. 1943).

\section{Resultados y discusión}

\section{Composición y estructura florística}

Riqueza florística. Se reportaron en las unidades de muestreo un total 924 individuos, de los cuales 818 se distribuyeron entre plántulas y juveniles, 106 individuos adultos con diámetros a $1,30 \mathrm{~m}$ de altura con DAP $\geq 10 \mathrm{~cm}$, todos discriminados en 14 familias, 16 géneros y 16 especies de plantas, siendo las más abundantes
Prioria copaifera Griseb. (68,8\%), seguida de Eschweilera garagarae Pitter (8,5\%), Leuchea seemanii $(7,6 \%)$, y Chrysophyllum caimito $(5,7 \%)$ (Figura 2).

Los resultados obtenidos en este estudio comparten muchos elementos florísticos con González etal. (1991), en un catival tipo A1 (los períodos de inundación oscilan entre 6 y 8 meses) la riqueza fue de 23,1 especies, mientras que en un catival tipo A2 (los períodos de inundación oscilan entre 3 y 6 meses) la riqueza fue de 43 especies. Por otro lado, en el Darién panameño en cativales dominados por $P$. copaifera, se encontró una riqueza de 86 especies (Grauel y Pineda 2001), aunque Golley et al. (1975) encontraron una diversidad menor (44 especies) en otro bosque de Panamá. No obstante, en algunos sitios, $C$. guianensis y $P$. officinalis son los únicos árboles asociados con cativo (Holdridge 1964, Espinal 1985). Además, en sitios donde la diversidad florística aumenta, se pueden encontrar otras especies maderables valiosas como: C. guianensis, Anacardium excelsum, Virola sp., Tabebuia rosea y Spondias mombin (Von Christen 1976).

\section{Análisis de la estructura poblacional}

Distribución horizontal. Los valores en DAP reportados en las unidades de muestreo arrojaron en total de 924 individuos (distribuidos en 386 para la UM1 y 538 en la UM2), donde 818 fueron plántulas (brinzales) y juveniles (latizales) 


\section{Bioetnia Volumen 14, 2017}

equivalente al $88,5 \%$ y adultos (fustales) con $\mathrm{DAP} \geq 10 \mathrm{~cm}$ que estuvieron integrados por 106 $(11,5 \%)$ individuos en donde las primeras tres clases diamétricas o intermedias que representan $88 \%$ de todos los individuos, proyectaron como resultado una distribución diamétrica con forma de campana de Gauss indicando un bosque con intervencion intermedia, el cual se encuentra en un estado avanzado de recuperación (Figura 3). Lo anterior se asemeja al estudio para la población de cativales definida por Mariscal et al. (2000), los cuales afirman que esta especie tiene una estructura diamétrica decreciente, con una marcada acumulación de individuos en las primeras categorías diamétricas y una disminución lenta a través de las clases diamétricas mayores es decir, que los individuos no son producto de una cohorte coetánea, y por tanto, existe una mezcla de edades (del Valle y Lema 1999). En este sentido González et al. (1991) y Linares (1988) reafirmaron que el grado de inundación al que está sometida la comunidad del catival, puede determinar sus características estructurales, pues ante una disminución del grado de inundación, la diversidad florística aumenta y el cativo deja de ser la especie dominante. De otro modo, Grauel (2004), encontró en un catival de Panamá que los árboles de un cm de DAP requieren 157 años para alcanzar $60 \mathrm{~cm}$ de DAP $(0,38 \mathrm{~cm}$ a menos de un cm año) y aproximadamente 186 años para llegar a $80 \mathrm{~cm}$ de DAP $(0,43 \mathrm{~cm}$ año). También del Valle (1979) empleó el método de tiempos de paso para calcular la curva de crecimiento del diámetro en función de la edad y encontró que el cativo tarda cerca de 100 años para alcanzar $60 \mathrm{~cm}$ de DAP, es decir, una tasa de crecimiento diamétrico de $0,6 \mathrm{~cm}$ año.

Distribucion vertical. De acuerdo con los resultados obtenidos sobre la distribución vertical y por clases diamétricas de los individuos y de las especies junto con lo observado en campo, se puede afirmar que el bosque estudiado posee tres estratos principales (disetaneo), discriminados así: 1) el estrato inferior o sotobosque, donde son muy abundantes las especies Anthurium sp., Psychotria $s p$. y Piper $s p$. que llega aproximadamente a 90 $\mathrm{cm}$ de altura (29,7\% del total de individuos). $A n$ thurium sp. en algunos sectores de la UM1 crecía formando densas agregaciones de vástagos, sobre todo en áreas con mayor penetración de luz solar, como consecuencia de la caída de árboles; 2) el estrato intermedio, donde es muy abundantes el género Miconia que alcanza aproximadamente 11 $m$ de altura (25,9\% del total); 3 ) el dosel o estrato superior conformado por árboles espaciados entre sí, el cual puede alcanzar aproximadamente hasta $24 \mathrm{~m}$ de altura y es un poco heterogéneo en cuanto a la composición de especies, pero en el
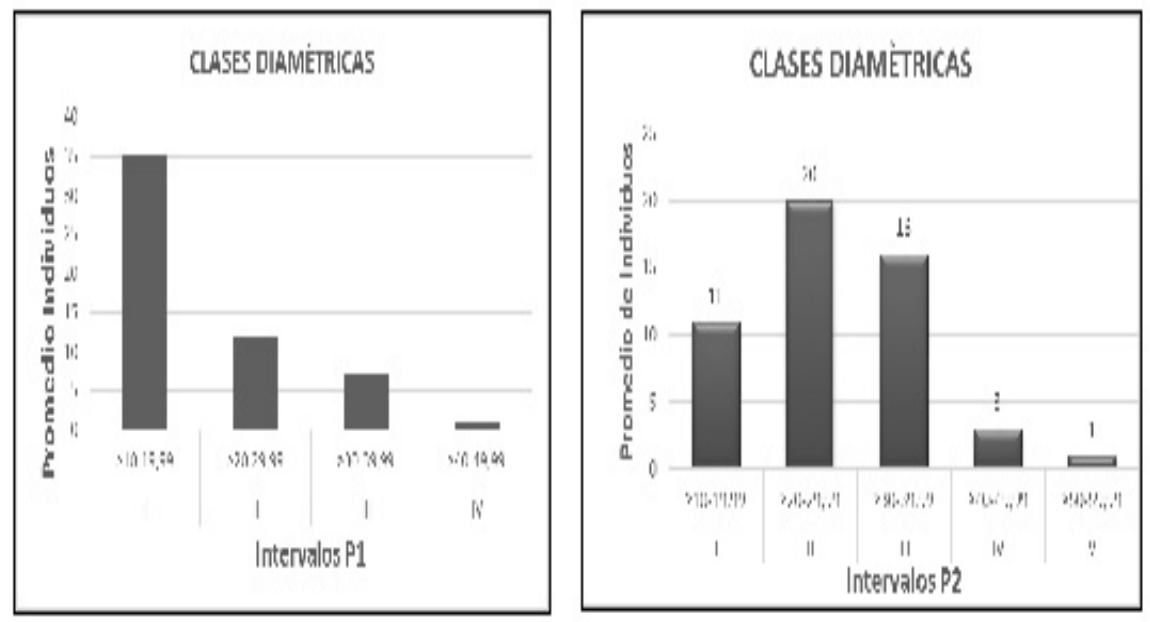

Figura 3. Clases diamétricas en las dos unidades de muestreo. 
que domina Prioria copaifera (44,2\% del total), siendo este estrato el que acumula la mayor área basal dentro de la comunidad (Figura 4).

Índices estructurales. Con base en el análisis elaborado para determinar el grado de importancia de las especies con mejor peso ecológico en las unidades de muestreo, se encontró que Prioria copaifera Griseb. con 69 individuos, obtuvo alrededor del $80 \%$ del IVI debido a su determinante área basal y su carácter gregario y dominante, la cual tiende a formar bosques monoespecíficos, donde su espesa copa no permite el desarrollo total de las especies establecidas bajo su cobertura, aunque cabe resaltar que otros especies también arrojaron un buen peso ecológico tales como Leuchea seemanii T \& P, Eschweilera sp., Spondias mombin L. y Grias cauliflora (Tabla 1). Diversidad de especies. De acuerdo con los

Tabla 1. Índice de valor de importancia en las unidades de muestreo (UM)

\begin{tabular}{|c|c|c|c|c|c|c|c|c|}
\hline \multirow{3}{*}{ Nombre científico } & \multicolumn{8}{|c|}{ Unidad de muestreo $\mathrm{N}^{\circ} 1$} \\
\hline & \multicolumn{2}{|c|}{ Abundancia } & \multicolumn{2}{|c|}{ Frecuencia } & \multicolumn{2}{|c|}{ Dominancia } & \multicolumn{2}{|c|}{ IVI al } \\
\hline & Abs. & Rel. & Abs. & Rel. & Abs. & Rel. & $300 \%$ & $100 \%$ \\
\hline Prioria copaifera Griseb. & 37 & 67,273 & 3 & 23,077 & 0,017 & 1,742 & 92,091 & 30,697 \\
\hline Leuchea seemanii T \& P & 4 & 7,273 & 2 & 15,385 & 0,358 & 35,776 & 58,433 & 19,478 \\
\hline Eschweilera sp. & 4 & 7,273 & 2 & 15,385 & 0,298 & 29,757 & 52,414 & 17,471 \\
\hline Morfoespecie 1 & 2 & 3,636 & 1 & 7,692 & 0,136 & 13,610 & 24,939 & 8,313 \\
\hline Chrysophyllum caimito & 3 & 5,455 & 1 & 7,692 & 0,118 & 11,775 & 24,922 & 8,307 \\
\hline Inga sp. & 2 & 3,636 & 2 & 15,385 & 0,017 & 1,742 & 20,763 & 6,921 \\
\hline Grias cauliflora & 2 & 3,636 & 1 & 7,692 & 0,042 & 4,161 & 15,489 & 5,163 \\
\hline Morfoespecie 2 & 1 & 1,818 & 1 & 7,692 & 0,014 & 1,439 & 10,950 & 3,650 \\
\hline Total & 55 & 100 & 13 & 100 & 1 & 100 & 300 & 100 \\
\hline
\end{tabular}

\begin{tabular}{|c|c|c|c|c|c|c|c|c|}
\hline \multirow{3}{*}{ Nombre científico } & \multicolumn{8}{|c|}{ Unidad de muestreo $\mathrm{N}^{\circ} 2$} \\
\hline & \multicolumn{2}{|c|}{ Abundancia } & \multicolumn{2}{|c|}{ Frecuencia } & \multicolumn{2}{|c|}{ Dominancia } & \multicolumn{2}{|c|}{ IVI al } \\
\hline & Abs. & Rel. & Abs. & Rel. & Abs. & Rel. & $300 \%$ & $100 \%$ \\
\hline Prioria copaifera Griseb. & 33 & 64,706 & 3 & 16,667 & 0,663 & 66,250 & 147,623 & 49,208 \\
\hline Spondias mombin L. & 1 & 1,961 & 1 & 5,556 & 0,181 & 18,110 & 25,627 & 8,542 \\
\hline Grias cauliflora & 3 & 5,882 & 2 & 11,111 & 0,021 & 2,125 & 19,119 & 6,373 \\
\hline Ochroma sp. & 2 & 3,922 & 2 & 11,111 & 0,007 & 0,746 & 15,778 & 5,259 \\
\hline Leuchea seemanii T \& P & 2 & 3,922 & 2 & 11,111 & 0,006 & 0,586 & 15,619 & 5,206 \\
\hline Eschweilera garagarae Pitter & 1 & 1,961 & 1 & 5,556 & 0,038 & 3,773 & 11,289 & 3,763 \\
\hline Pentaclethra macroloba & 2 & 3,922 & 1 & 5,556 & 0,017 & 1,679 & 11,156 & 3,719 \\
\hline Morfoespecie 1 & 2 & 3,922 & 1 & 5,556 & 0,014 & 1,365 & 10,842 & 3,614 \\
\hline Morfoespecie 2 & 1 & 1,961 & 1 & 5,556 & 0,019 & 1,925 & 9,441 & 3,147 \\
\hline Tabebuia rosea & 1 & 1,961 & 1 & 5,556 & 0,015 & 1,457 & 8,973 & 2,991 \\
\hline Castilloa elastica Cerv. & 1 & 1,961 & 1 & 5,556 & 0,012 & 1,171 & 8,687 & 2,896 \\
\hline Inga sp. & 1 & 1,961 & 1 & 5,556 & 0,006 & 0,604 & 8,120 & 2,707 \\
\hline Sapium caudatum Pittier & 1 & 1,961 & 1 & 5,556 & 0,002 & 0,210 & 7,726 & 2,575 \\
\hline Total & 51 & 100 & 18 & 100 & 1 & 100 & 300 & 100 \\
\hline
\end{tabular}

Abs. $=$ absoluta $\quad$ Rel. $=$ Relativa 


\section{Bioetnia Volumen 14, 2017}
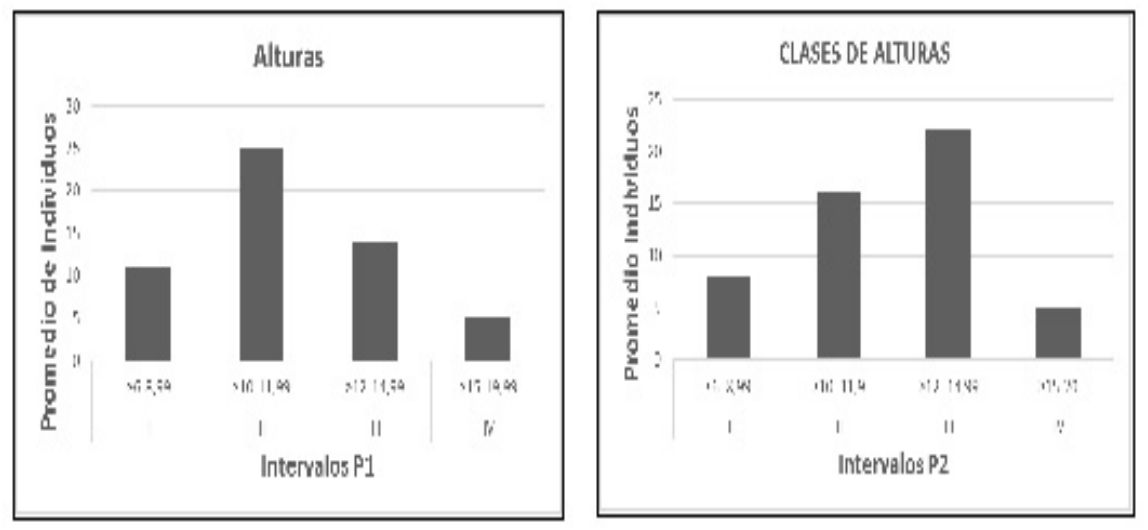

Figura 4. Categorías altitudinales en las unidades de muestreo.

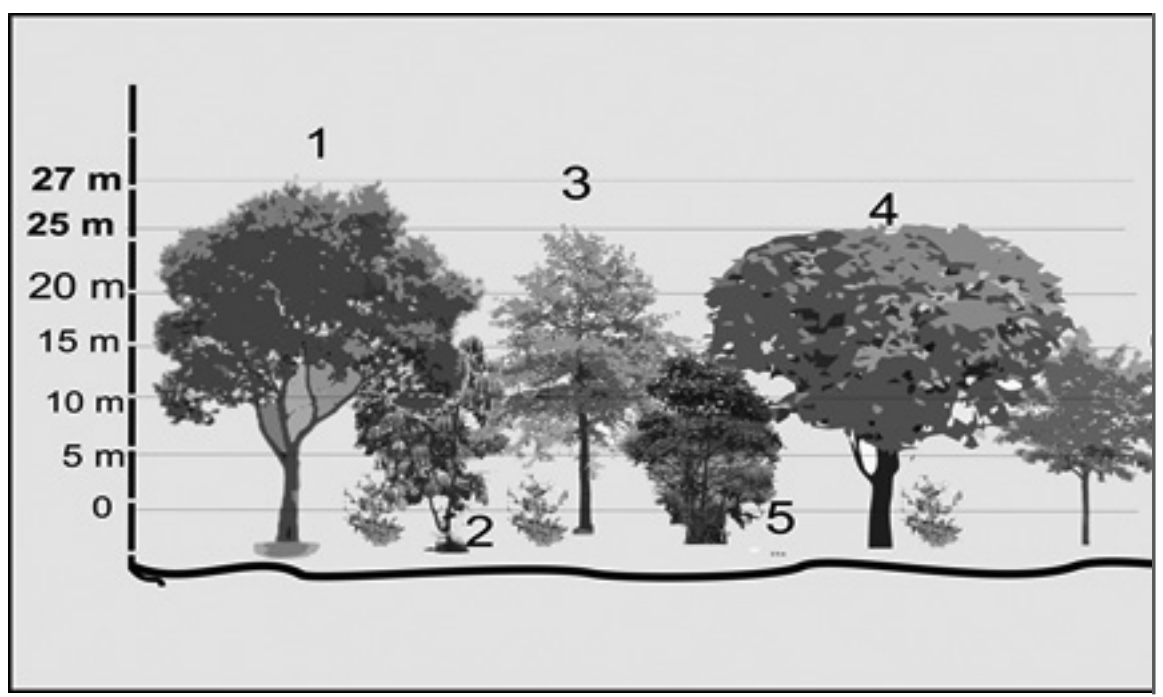

Figura 5. Perfil idealizado de la asociacion de cativales. 1. Prioria copaifera Griseb. 2. Pentaclethra macroloba. 3. Cecropia sp. 4. Inga spp. 5. Grias cauliflora. 6. Leuchea seemanii T \& P.

Tabla 2. Valores de diversidad en las unidades de muestreo (UM)

\begin{tabular}{lrr}
\hline \multicolumn{1}{c}{ Diversidad } & UM 1 & UM 2 \\
\hline N $^{\circ}$ Individuos & 386 & 538 \\
Riqueza & 8 & 13 \\
Shannon & 1,241 & 1,496 \\
Simpson & 0,539 & 0,420 \\
Equidad & 0,597 & 0,583 \\
Margalef & 1,175 & 1,908 \\
\hline
\end{tabular}

índices de Margalef, Shannon y riqueza, la diversidad de especies fue significativamente menor en la UM 1; en contraste, el índice de Simpson no mostró diferencias significativas entre las UM. Esta discreta diversidad puede atribuirse a condiciones físicas como la topografía variada, actividades humanas y las condiciones del terreno, los cuales de acuerdo con García-Montiel (2002), se debe tomar en cuenta el efecto de las perturbaciones humanas principalmente a la realización de actividades con fines ganaderos (p.e., uso del fuego) y extracción ilegal de madera (p.e., árboles grandes de especies valiosas).

Gremios de especies asociadas con los cativales. La asociación de cativales estudiada en inmediaciones del río León, está conformada de acuerdo con Finegan (1993), por varios grupos ecológicos (heliófitas efímeras, heliófitas durables, esciófitas parciales y esciófitas totales), los cuales reúnen especies arbóreas de similares características biológicas y ecológicas, que comparten patrones de regeneración natural, potencial de crecimiento, propiedades de la madera y tipos de usos (Figura 5). Muchas de las especies vegetales que 
integran los cativales tienen germinación epigea (P. copaifera, Virola sebifera, Otoba gracilipes, Brosimun sp.) y con la notable disponibilidad de reservas alcanzan crecimientos enormes en la etapa de brinzal (p.e., P. copaifera, C. guianensis, Ochroma sp.). De otro lado, estas especies ofrecen diversos nichos y medios alimenticios a innumerables individuos que conforman la fauna silvestre, especialmente roedores, que llegan a consumir cantidades significativas de semillas (C. guianensis), lo que sirve como mecanismo de dispersión, pues en épocas de abundancia ciertos animales como la guagua (Agouti paca), transportan y entierran estas semillas; algunas escapan del consumo logrando germinar y así ayudan a la repoblación del catival.

\section{Consideraciones finales}

Según los índices de Margalef, Shannon y riqueza, la diversidad de especies fue significativamente menor en la UM 1; en contraste, el índice de Simpson no mostró diferencias significativas entre las UM. Esta discreta diversidad se puede atribuir a condiciones físicas como la topografía variada, actividades humanas y condiciones del terreno.

Con base en el análisis elaborado para determinar el grado de importancia de las especies con mejor peso ecológico en las unidades de muestreo se encontró que Prioria copaifera Griseb. con 69 individuos, obtuvo alrededor del 80\% del IVI, debido a su determinante área basal y su carácter gregario y dominante, la cual tiende a formar bosques monoespecíficos

Muchas de las especies vegetales integran los cativales como Virola sebifera, Otoba gracilipes, Brosimun sp., C. guianensis, Ochromasp.; se hace necesario establecer programas de restauración ecológica urgente con el objetivo de aumentar las poblaciones que sin duda han sido diezmadas por las actividades antrópicas imperantes en la zona dentro de este ecosistema singular.
Los bosques de cativo agrupan su mayor área basal en las últimas clases diamétricas, donde existen pocos individuos y especies, pero de grandes dimensiones. La distribución de los árboles y número de especies por clase diamétrica tomaron forma de "J" invertida.

\section{Literatura citada}

Curtis JT, McIntosh RP. 1951. An upland forest continuum in the prairie-forest border region of Wisconsin. Ecology. 32 (3): 476-96. Disponible en: https://www.jstor. org/stable/1931725? seq=1\#page_scan tab_contents

Dallmeier F (ed.). 1992. Long-term monitoring of biological diversity in tropical forest areas: methods for establishment and inventory of permanent plots. Paris: UNESCO; 73 pp. Disponible en: http://unesdoc.unesco. org/images/0009/000938/093876eo.pdf

Dawkins HC. 1958. The management of natural tropical high-forest with special reference to Uganda. Oxford: Imperial Forestry Institute, University of Oxford; 155 pp.

Del Valle JI, Lema A. 1999. Crecimiento de cohortes de árboles coetáneos en rodales espacialmente dispersos: el caso de Campnosperma panamensis. Acad Colomb Cienc. 23: 249-60.

Del Valle JI. 1979. Curva preliminar de crecimiento del cativo (Prioria copaifera) en bosque virgen empleando el método de los tiempos de paso. Fac Nal Agr. (32): 19-26.

Del Valle JI. 2005. Crecimiento diamétrico de árboles de humedales del Pacífico colombiano. Bogotá: Interciencia

Duivenvoorden JF. 1996. Patterns of tree species richness in rain forests of the middle Caquetá Area, Colombia, NW Amazonia. Biotropica. 28 (2): 142-58. Disponible en: https://www.jstor.org/stable/2389070?seq=1\#page scan tab_contents

Escobar JA. 1987. Caracterización de tipos de cativales. Tesis de pregrado. Medellín: Facultad de Ciencias Agropecuarias, Universidad Nacional de Colombia; 198 pp.

Espinal LS. 1985. Geografía ecológica del departamento de Antioquia (zonas de vida formaciones vegetales del departamento de Antioquia). Rev Fac Nal Agr. 38 (1): 5-106.

Forero E, Gentry A. 1989. Lista anotada de las plantas del departamento del Chocó, Colombia. Bogotá: Biblioteca José Jerónimo Triana $\mathrm{N}^{\circ} 10$, Instituto de Ciencias Naturales, Universidad Nacional de Colombia.

Finegan B. 1993. Curso intensivo internacional de silvicultura y manejo de bosques naturales tropicales. I. 


\section{Bioetnia Volumen 14, 2017}

Tema: bases ecológicas para la silvicultura. Turrialba: Catie; 225 pp.

Fisher RA, Cobet AS, Williams CB. 1943. The relation between the number of species and the number of individuals in a random sample of an animal population. J Anim Ecol. 12: 42-58. Disponible en: https://www. jstor.org/stable/1411?seq=1\#page scan tab contents

García-Montiel DC. 2002 El legado de la actividad humana en los bosques neotropicales contemporáneos. En: Guariguata MR, Kattan GH(eds). Ecología y conservación debosques neotropicales. Cartago: Libro Universitario Regional (EULAC-GTZ); pp. 97-116.

Gentry A. 1982. Phytogeographic patterns as evidence for a Chocó refuge. In: Prance GT (ed.). Biological diversification in the tropics. New York: Columbia University Press; pp. 112-36.

Golley FB, McGinnis JT, Clements RG, Child GI, Duever MJ. 1975. Mineral cycling in a tropical moist forest ecosystem. Athens: University of Georgia Press; 248 pp.

González H, Gómez HD, Arteaga FJ. 1991. Aspectos estructurales de un bosque de cativo en la región del bajo Atrato, Colombia. Rev Fac Nal Agr. 44 (1/2): 3-50.

Grauel WT, Pineda RM. 2001. Manual técnico para el manejo sostenible de los cativales en Darién, Panamá. Panamá: Autoridad Nacional del Ambiente, Smithsonian Tropical Research Institute. Disponible en: $\underline{\text { https:// }}$ searchworks.stanford.edu/view/5050276

Grauel WT. 2004. Ecology and management of wetland forests dominated by Prioria copaifera in Darien, Panamá. PhD Thesis. Gainesville: University of Florida; 178 pp.

Greig Smith P. 1983. Quantitative plant ecology. $3^{\text {er }}$ ed. Berkeley: University of California Press; 359 pp.

Golley FB, McGinnis JT, Clements RG, Duever MJ. 1969. The structure of tropical forest in Panama and Colombia. Bioscience. 19: 693-7.

Hart TB. 1990. Monospecific dominance in tropical rain forest. Trends Ecol Evol. 5 (1): 6-10. Disponible en: https://www.ncbi.nlm.nih.gov/pubmed/21232309

Holdridge LR. 1964. Ecology. En: Wilson NC. (ed.). El real environmental survey. Darien Province:US Army Transportation Research Command; 355 pp.

Hubbell SP, Foster RB. 1983. Diversity of canopy trees in a neotropical forest and implications for conservation. pp. 25-41.En: Sutton SL, Whitmore TC, ChadwickAC (eds.). Tropical rain forest: Ecology and management. Oxford: Blackwell Scientific.

Holdridge LR. 1964. Ecology. En: Wilson NC (ed.). El real environmental survey; $355 \mathrm{pp}$.

Holdridge L. 1979. Ecología basada en zonas de vida. Serie de libros y materiales educativos $\mathrm{N}^{\circ} 34$. San José: IICI; 216 pp
Igac, Inderena, Conif. 1984. Mapa de bosques de Colombia. Memoria explicativa. Bogotá: Igac, Inderena, Conif; 206 pp.

Linares R. 1988. Estudio preliminar de la asociación catival en Colombia. Serie documentación 17. Bogotá: Corporación Nacional de Investigación y Fomento Forestal (CONIF); 78 pp.

Lot A, Chiang F. 1986. Manual de herbario: Administración y manejo de colecciones, técnicas de recolección y preparación de ejemplares botánicos. México DF: Consejo Nacional de Ciencia y Tecnología; 142 pp.

López R, Montero I. 2006. Prioria copaifera. En: Cárdenas D, Salinas NR (eds.). Libro rojo de plantas de Colombia. Especies maderables amenazadas. Bogotá: Instituto Amazónico de Investigaciones (SINCHI), Ministerio de Ambiente, Vivienda y Desarrollo Territorial; 169 pp. Disponible en: http://observatorio.epacartagena. gov.co/wp-content/uploads/2016/12/LibroRojoMaderables.pdf

López OR, Kursar TA. 2007. Interannual variation in rainfall, drought stress and seedling mortality may mediate monodominance in tropical? Ooded forests. Oecologia. 1: 35-43.

MargalefR. 1977. Ecología. Barcelona: Ediciones Omega; $951 \mathrm{pp}$.

Mariscal E, Martínez R, Takano K. 2000. Manejo de bosques naturales. Estudios, ensayos y demostraciones. Ciudad de Panamá: Proyecto de Desarrollo Técnico de la Conservación de los Bosques (CEMARE), Autoridad Nacional del Ambiente de Panamá (ANAM), Agencia de Cooperación Internacional del Japón (JICA); 138 pp.

Rangel-Ch JO, Lowy-CP. 1993. Tipos de vegetación y rasgos fitogeográficos en la región pacífica de Colombia. En: Leyva P. (ed.). Colombia Pacífico. Tomo I. Bogotá: Fondo FEN.

Richards PW. 1996. The tropical rain forest: an ecological study. $2^{\text {nd }}$ ed. Londres: Cambridge University Press; $600 \mathrm{pp}$.

Suárez AE, Hurtado G, Carvajal FG, Rodríguez JE, RodríguezR. 1984. Mapa de bosques de Colombia: memoria explicativa. Bogotá: Instituto Geográfico Agustín Codazzi (IGAC), Instituto de los Recursos Naturales y delAmbiente (INDERENA) y Corporación Nacional de Investigación y Fomento Forestal (CONIF); 206 pp.

Von Christen HC. 1976. Clasificación preliminar y evaluación de los sitios de la tierra baja húmeda de Colombia para el manejo forestal, con especial consideración de los suelos hidromórficos. En: Frankfurt SE (ed.). Enfoques colombianos, ecología, suelos del trópico. Monografías $N^{\circ}$ 8. Bogotá: Fundación F. Naumann; pp. 9-103. 